This manuscript was accepted by Rev. Sci.Instrum. Click here to see the version of record.

\title{
$24 \mathrm{~mJ} \mathrm{Cr} \mathbf{r}^{+4}$ :forsterite four-stage master-oscillator power-amplifier laser system for high resolution mid-infrared spectroscopy
}

\author{
Lyubomir I. Stoychev ${ }^{1,2,3}$, Humberto Cabrera ${ }^{1,3, a)}$, Komlan S. Gadedjisso-Tossou ${ }^{1,3,4}$, Nikolai Vasiliev ${ }^{5}$, Yuri \\ Zaporozhchenko $^{5}$, Ivaylo P. Nikolov ${ }^{6}$, Paolo Sigalotti ${ }^{6}$, Alexander A. Demidovich ${ }^{6}$, José J. Suárez-Vargas ${ }^{1,3}$, Emiliano \\ Mocchiutti $^{1}$, Cecilia Pizzolotto ${ }^{1}$, Joseph Niemela $^{3}$, Marco Baruzzo ${ }^{1,7}$, Miltcho B. Danailov ${ }^{6}$ and Andrea Vacchi ${ }^{1,7}$ \\ ${ }^{1}$ Istituto Nazionale di Fisica Nucleare, Sezione di Trieste, Trieste, 34149, Italy \\ ${ }^{2}$ Institute of Solid State Physics, Bulgarian Academy of Sciences, 72 Tzarigradsko Chaussee, Sofia 1784, Bulgaria \\ ${ }^{3}$ Optics Lab., The Abdus Salam International Centre for Theoretical Physics, Trieste, 34151, Italy \\ ${ }^{4}$ Laboratoire de Physique des Composants à Semi-conducteurs (LPCS), Université de Lomé, 01 BP 1515 Lomé, Togo \\ 5"LOTIS TII" Ltd, 17, Oktyabrskaya str., Minsk 220030, Belarus \\ ${ }^{6}$ Elettra-Sincrotrone, S.C.P.A, Trieste 34149, Italy \\ ${ }^{7}$ Mathematics and Informatics Department, Udine University, Udine 33100, Italy
}

We present the design of a Cr:forsterite based single-frequency master-oscillator power-amplifier (MOPA) laser system delivering much higher output energy compared to previous literature reports. The system has four amplifying stages with two-pass configuration each, thus enabling the generation of $24 \mathrm{~mJ}$ output energy in the spectral region around $1262 \mathrm{~nm}$. It is demonstrated that the presented Cr:forsterite amplifier preserves high spectral and pulse quality allowing a straightforward energy scaling. This laser system is a promising tool for tunable nonlinear down-conversion to the midinfrared (MIR) spectral range, and will be a key building block in a system for high-resolution muonic hydrogen spectroscopy in the 6.8 micron range.

a) Author to whom correspondence should be addressed: Humberto.Cabrera@ts.infn.it; hcabrera@ictp.it

\section{INTRODUCTION}

High laser pulse energy is an essential parameter to excite any atomic or molecular system with low transition probability as well as to produce high efficiency nonlinear down-conversion to the nearinfrared (NIR) and MIR spectral range. MIR lasers and photonic technologies have been developed in the past years, but technical challenges have slowed down the development of convenient instrumentation in this spectral region. An NIR tunable narrow-bandwidth high energy laser source is potentially very interesting source for high resolution spectroscopy, because by using difference frequency generation (DFG) with a broadly available fixed-wavelength Nd:YAG lasers, its radiation can be efficiently down-converted to the MIR. ${ }^{1}$ The motivation behind our work was to generate tunable radiation useful for muonic hydrogen atom spectroscopy. ${ }^{2,3}$ In this direction, the final target is the measurement of the hyperfine splitting of the $\mu \mathrm{p}$ atom in the ground state $1 \mathrm{~S}$ by using a pulsed laser source with high energy output $(>1.5 \mathrm{~mJ})$, tunable at wavelength around $6785 \mathrm{~nm}$ with a narrow linewidth $(<70 \mathrm{pm})$ enabling the transition of interest with a sufficient probability. ${ }^{2,3}$

These above mentioned parameters can be achieved using a configuration based on direct DFG in non-oxide crystal, cut for type II non-linear process, with pump and signal coming from one narrowband fixed wavelength and one tunable solid state lasers. This solution is based on mixing single frequency (SF) Nd:YAG laser $(\lambda=1064 \mathrm{~nm})$ and a tunable SF narrow bandwidth, Cr:forsterite laser $(\lambda \approx 1262 \mathrm{~nm})$ pumped by a second Nd:YAG synchronized to the first one. 
This manuscript was accepted by Rev. Sci.Instrum. Click here to see the version of record.

Publishing lowever, the desired output energy $(>1.5 \mathrm{~mJ})$ can be reached if the energy at $1262 \mathrm{~nm}$ radiation exceeds $20 \mathrm{~mJ}$. The most common lasers that satisfy these requirements are based on Cr:forsterite active medium, which produce SF tunable NIR radiation $(1130-1367 \mathrm{~nm}){ }^{4}$ However, the reported output energies from Cr:forsterite lasers typically do not exceed few $\mathrm{mJ}^{5}{ }^{5}$

Often the most common approach to increase the output pulse energy of a laser is to add one or more amplifying stages to the laser system. Amplification of pulsed or continuously wave power from an oscillator can be achieved using a MOPA configuration. ${ }^{6}$ A common arrangement for multi-pass amplification uses an array of mirrors to reflect the pulse repeatedly through the gain medium. Most recently, this approach was implemented in a Cr:forsterite amplifier in order to reach $10.8 \mathrm{~mJ}{ }^{7}$ Therefore, it was demonstrated that, increasing either the number of stages or the number of passes the extraction efficiency can be improved. However, the efficiency can be significantly enhanced if stage and passage number increase simultaneously. ${ }^{8} \mathrm{~A}$ step in this direction is the implementation of a four-stage amplifier with the two-pass configuration each to increase the extraction efficiency and the output energy. ${ }^{6}$

In this work, we report a SF Cr:forsterite four-stage MOPA laser system with two-pass per stage which delivers up to $24 \mathrm{~mJ}$, starting from a relatively low energy of the oscillator and without significantly affecting the beam parameters. The achieved energy is the highest value ever reported in SF Cr:forsterite laser in the literature.

\section{EXPERIMENTAL SETUP}

\section{Laser system}

The laser system is composed of two blocks as follows: the SF Cr:forsterite oscillator and the multipass amplifier unit.

Laser oscillator block. A simplified scheme of the laser oscillator is shown in Fig. 1. It is an integrated head that includes a Nd:YAG pump laser $(25 \mathrm{~Hz}, 100 \mathrm{~mJ}$, TEM00 mode, $10 \mathrm{~ns})$ and a tunable SF Cr:forsterite laser (désigned by LOTIS TII) with crystals (Solix Ltd) cut at Brewster angle, both for the oscillator and the amplifiers' stages. The Brewster-angled crystals, with two different dimensions $(9 \times 15 \times 16 \mathrm{~mm}, 9 \times 15 \times 20 \mathrm{~mm}, 9 \times 15 \times 20 \mathrm{~mm}, 9 \times 15 \times 16 \mathrm{~mm})$ were cut for minimum losses of $b$-axis polarized radiation. Therefore, the Cr:forsterite crystal was oriented such that the $b$-axis polarized emission was $p$ polarized and the radiation propagates along the c-axis. The absorption coefficient of the crystals is $\sim 1.5 \mathrm{~cm}^{-1}(1064 \mathrm{~nm})$ and the figure of merit FOM $=\alpha(1064 \mathrm{~nm}) / \alpha(1262 \mathrm{n}$ $\mathrm{m}) \geq 25$, where $\alpha$ is the absorption coefficient at the proper wavelength. Wavelength selection is provided by the holographic diffraction grating (Spectrogon, 1350 grooves $/ \mathrm{mm}$ ) set at incidence angle $\sim 83^{\circ}$.

The pump radiation is directed to the Cr:forsterite laser cavity by two turning mirrors (15) and a polarization attenuator consisting of a retarding plate $\lambda / 2$ (17) and a polarizer (18). The attenuator is designed for smooth adjustment of the pump radiation without changing the parameters of the $\mathrm{Nd}$ : YAG laser. The required excitation energy density in the resonator is created by the decreasing telescope (20). The intracavity telescope (4) together with the pinhole (2) provide near TEM $\mathrm{T}_{00}$ mode operation of the pump laser.

Input and output mirrors (21) and (25) of the Cr:forsterite cavity are set in Thorlabs Polaris kinematic mounts. The input mirror mount (21) was placed on the NFL5DP20S/M closed-loop piezo stage which have a $20 \mu \mathrm{m}$ piezo travel with $0.6 \mathrm{~nm}$ resolution when driven by one of the Thorlabs K-Cube KPZ101, 0 to $75 \mathrm{~V}$, piezo controller. This configuration provides fine adjustment of the cavity and maintains SF operation of the selected lasing wavelength. 


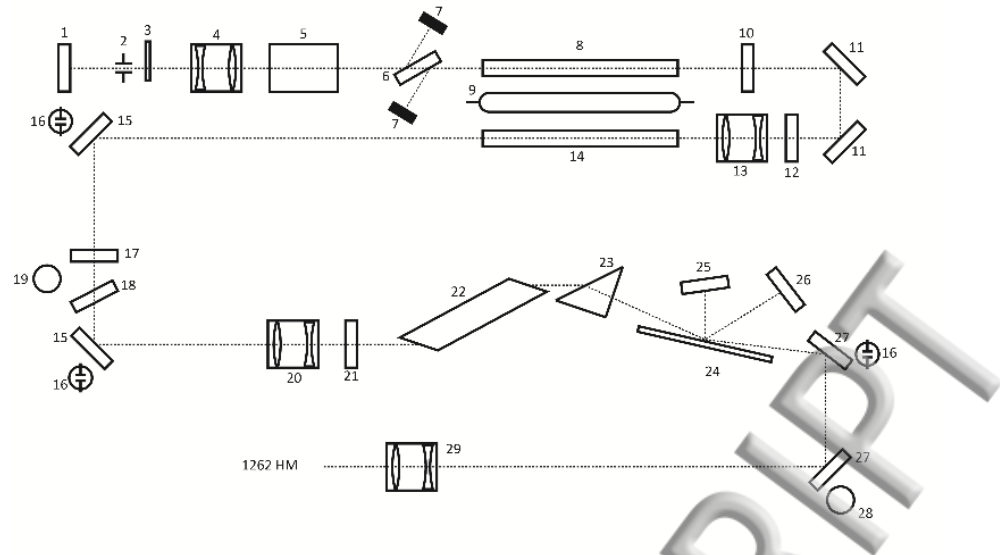

FIG. 1. Schematic diagram of the laser oscillator. 1: rear mirror of the pump resonator, 2: intracavity pinhole, 3: intracavity shutter, 4: intracavity telescope, 5: Pockels cell (KDP), 6: polarizer, 7: beam stops , 8: active element (AE) of the master laser (4×80 mm), 9: pump lamp, 10: output mirror $(1064 \mathrm{~nm}), 11$ : turning mirrors $(1064 \mathrm{~nm}), 12$ : rotator $90^{\circ}(1064 \mathrm{~nm}), 13$ : increasing telescope, 14: AE amplifier $(5 \times 80 \mathrm{~mm}), 15$ : turning mirrors $(1064 \mathrm{~nm}), 16$ : photoprobes, 17: half-wave plate $(1064 \mathrm{~nm}), 18$ : polarizer, 19: beam stop, 20: decreasing telescope, 21: input cavity mirror (1257-1267 nm), 22: Cr:forsterite AE, 23: right-angle prism (STK19 glass), 24: diffraction grating, 25: rear mirror (1257-1267 nm), 26: mirror (1257-1267 nm), 27: mirrors (1257-1267 nm), 28: beam stop, 29: increasing telescope.

The rear mirror (25) is set in a Polaris-K1 holder with 3 adjusters (Thorlabs). The right-angle prism (23) provides a coarse setting of the output wavelength (pre-selector), increased homogeneity of the pump distribution in the active element (AE) and protection of the diffraction grating (24) from high intensity of the pump radiation $(1064 \mathrm{~nm})$ due to the reflection of the pump light that has passed through the $\mathrm{AE}$ from the face of the prism cathetus. The coating at this prism edge reflects the 1064 $\mathrm{nm}$ light and fully transmits the Cr:forsterite radiation $(1257-1267 \mathrm{~nm})$. All optical elements are mounted on honeycomb stainless breadboard $\sim 770 \times 360 \times 70 \mathrm{~mm}$. The control and alignment of the resonator SF mode is performed using the oscillograms obtained from the built-in fast IR photodetector (16) (photodiode FGA01).

An increase in the temperature of the Cr:forsterite crystal significantly reduces the generation efficiency. ${ }^{9}$ Therefore, to increase the lasing efficiency and increase the stability of the generation parameters, the $\mathrm{Cr}$ :forsterite crystal holder is cooled with a thermoelectric cooling system; the coolant temperature was set to $-12^{\circ} \mathrm{C}$ (approximately $1-2^{\circ}$ above the dew point).

A common method of narrowing the bandwidth of a pulsed laser is to use the grazing incidence cavity (GIC). ${ }^{10}$ But this design of laser cavity is not enough for Cr:forsterite stable operation in SF especially with prr $>10 \mathrm{~Hz}$. One of the effective way to realize SF operation is to use a multi-arm grazing incidence coupled cavity (Fox-Smith coupled cavity). ${ }^{11}$ This cavity has better mode selection than the conventional GIC and operates at a lower threshold. A conventional GIC was created between the mirrors (21) and (25) via a first order diffraction from the grating, with the zero order diffraction forming the output. In the case of the Fox-Smith coupled cavity not all the light reflected from mirror (25) is returned directly to the gain medium via diffraction in the grating. Some of this light is instead reflected from the grating towards mirror (26) before being returned to the grating. During operation, a fraction of the first order diffracted light will always remain between mirror (25), the grating and mirror (26), making multiple round trips. The mirror (26) was mounted on a kinematic mirror mount (POLARIS-K1S3P) with 3 piezoelectric adjusters for fine aligning as well as precise control of the additional cavity length. The addition of the extra mirror (26) has two benefits: light that would otherwise be lost from the cavity is retained which leads to both a reduction in lasing threshold and the formation of a Fox-Smith interferometer. In this interferometer, the grating performs the role of the beam-splitter with the zeroth order diffraction corresponding to the output beam and the first order reflected to the mirrors. At first, we adjusted the $\mathrm{Cr}$ :forsterite cavity length for receiving SF operation using the piezo stage in the rear mirror (25). In SF the laser pulse has a smooth form without mode beatings. Then we installed the additional mirror (26), whose position was adjusted. Stable SF 
Publishingoperation of the Cr:fosterite oscillator with $1.5 \mathrm{~mJ}$ energy, with a stability of $2 \%$ RMS, was observed at pump energy of $40 \mathrm{~mJ}$. The optimal angle of incidence on the grating was $\sim 83^{\circ}$.

Multi-pass amplifying block. The optical scheme of the amplifying block is shown in Fig. 2 and is composed of four amplifying stages pumped by two pulsed Q-switched Nd:YAG lasers: Spitlight 600 max energy $600 \mathrm{~mJ}$ ) from Innolas and LS-2138 (max energy $220 \mathrm{~mJ}$ ) from LOTIS TII, both lasing at fundamental frequency $(1064 \mathrm{~nm})$. Pumping is set longitudinal, with an angle of less than $3^{\circ}$ between the pump and the Cr:forsterite signal on each pass. Each stage includes a Brewster-cut crystal and works in two-pass configuration. The energy of the pump is distributed between the stages by half-wave plates (1) and polarizers (2). An external polarizer, not shown in the figure, eliminates the depolarized component of the pump laser radiation and transmits only the laser radiation with horizontal polarization. Matching between Cr:forsterite amplifying and pump beams is guaranteed by the telescopes (3) and (6).

The output energies of the lasers were measured by J-25MB-HE and EnergyMax-USB J-50MB-YAG energy sensors (Coherent), while the pulse shapes were taken by an InGaAs pin-photodiode detector (ET-3010, Electro-Optics Technology, Inc) and recorded with a $2 \mathrm{GHz}$ oscilloscope (Lecroy WaveRunner G 6200A).

FIG. 2. Schematic diagram of the multi-stages amplifier. 1: half-wave plates (1064 nm), 2: polarizers, 3: compressing telescopes (1064 $\mathrm{nm}), 4$ : turning mirrors $(1262 \mathrm{~nm}), 5$ : Cr:forsterite active element, 6: expanding telescopes (1225-1275 nm), 8: beam stops.

\section{RESULTS AND DISCUSSION}

The optimum time delay between the pulses of the pump and the Cr:forsterite oscillator beams was $25 \mathrm{~ns}$. The starting energy of the Cr:forsterite oscillator was $1.5 \mathrm{~mJ}$ and the diameters of the pump and Cr:forsterite beams were optimized to achieve the maximum energy extraction.

For the first stage of amplification, the pump beam of diameter $\varnothing=6 \mathrm{~mm}$ was compressed to $\varnothing=3$ $\mathrm{mm}$ using the telescope $3\left(2^{\mathrm{x}}\right)$, which for the pump energy of $85 \mathrm{~mJ}$ provides the optimum excitation energy density of $1.2 \mathrm{~J} / \mathrm{cm}^{2}$ in the crystal. The input diameter of the Cr:forsterite beam was expanded to $\varnothing=3 \mathrm{~mm}$ using the telescope $6\left(1.8^{\mathrm{x}}\right)$ given an energy density $0.021 \mathrm{~J} / \mathrm{cm}^{2}$ at the input of the first crystal. For the second amplification stage, the pump beam $(\varnothing=6 \mathrm{~mm})$ was compressed to $\varnothing=4.5$ $\mathrm{mm}$ using the telescope $3\left(1.3^{\mathrm{x}}\right)$, resulting in an energy density of $0.94 \mathrm{~J} / \mathrm{cm}^{2}$ for the pulse energy of $150 \mathrm{~mJ}$. Additionally, the Cr:fosterite spot was expanded to a diameter of $\varnothing=4.2 \mathrm{~mm}$ by the telescope $6\left(1.4^{x}\right)$, corresponding to the energy density of $0.019 \mathrm{~J} / \mathrm{cm}^{2}$ at the input of the second amplifying stage, thus giving $2.7 \mathrm{~mJ}$ output energy. For the third stage, the pump beam spot was increased to $\varnothing$ $\sim 5.4 \mathrm{~mm}$ and the energy was $175 \mathrm{~mJ}$ (energy density of $0.77 \mathrm{~J} / \mathrm{cm}^{2}$ ) what produced $13 \mathrm{~mJ}$ output energy. Finally, for the fourth stage the pump beam spot $(\varnothing=5.4 \mathrm{~mm})$ was increased to $\varnothing \sim 6 \mathrm{~mm}$ 
Publishingvith the $190 \mathrm{~mJ}$ energy (energy density value of $0.67 \mathrm{~J} / \mathrm{cm}^{2}$ ) to convert the output amplifier energy to $24 \mathrm{~mJ}$.

The obtained output energy $(1262 \mathrm{~nm})$ before and after each stage of the amplifier, as well as the pump beam energy density used are shown in Table I. As it can be seen, the starting oscillator input energy of $1.5 \mathrm{~mJ}$ reached $24 \mathrm{~mJ}$ after the fourth amplification stage, corresponding to an overall gain of 16. This corresponds to an average gain of 2 per pass. The gain was defined as the amplifier output energy divided by the energy from the oscillator at the input of the first stage. The gain per stage varies both due to different sizes of the Cr:forsterite crystals and to the different pump energy densities. In general, the gain value agrees quite well with the experimental values previously measured using similar configurations. ${ }^{7,10}$ However, the expected gain, for the two crystals lengths, oscillates between 3.2 and 4 according to the calculations performed before. ${ }^{7,10}$ The gain measured experimentally is lower than the expected theoretical one because the theory does not take into account the contribution to the gain reduction caused by the effects of excited-state absorption, passive loss in the rod as well as local heating and thermal lensing. ${ }^{10}$

\begin{tabular}{|c|c|c|c|}
\hline N stages & $E_{\text {in }}(1262 \mathrm{~nm}) \mathrm{mJ}$ & $\begin{array}{c}\mathrm{E}_{\text {out }}(1262 \mathrm{~nm}) \\
\mathrm{mJ}\end{array}$ & $\mathrm{E}_{\mathrm{d}}(1064 \mathrm{~nm}) \mathrm{J} / \mathrm{cm}^{2}$ \\
\hline 1 & 1.50 & 2.70 & 1.20 \\
2 & 2.70 & 5.50 & 0.94 \\
3 & 5.50 & 13 & 0.88 \\
4 & 13 & 24 & 0.70 \\
\hline
\end{tabular}

TABLE I. Energy values before $E_{\text {in }}$ and after $E_{\text {out }}$ the forsterite amplifier and pump energy density $E_{d}$ per step.

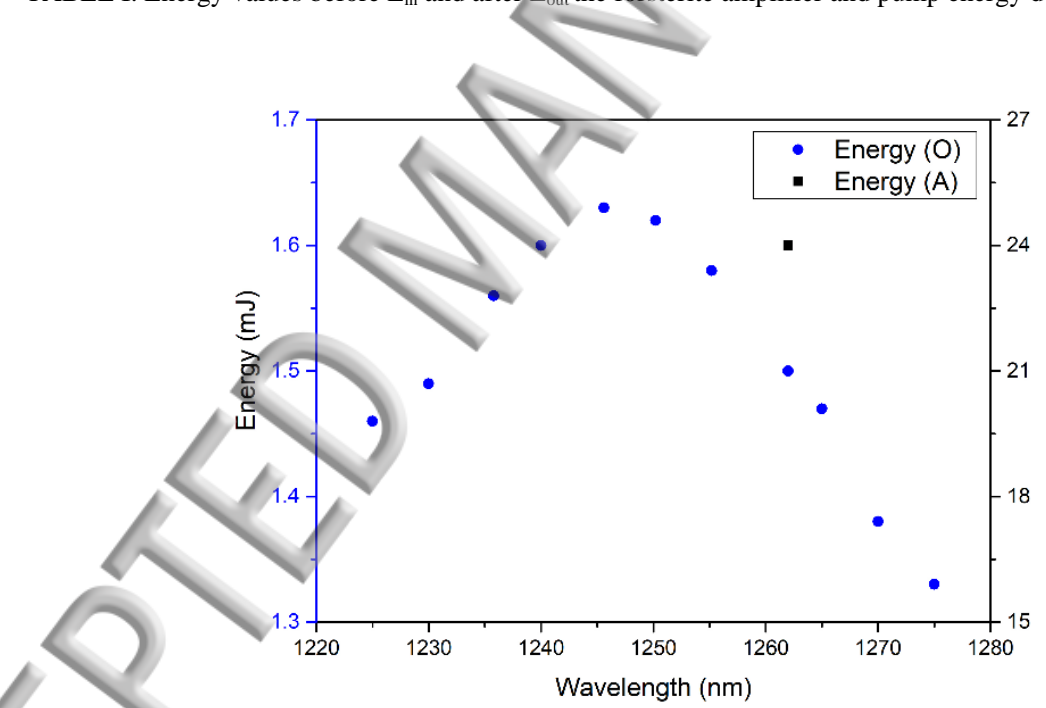

FIG. 3. Energy output versus the wavelength of the single-mode Cr:forsterite oscillator (O) and amplifier (A).

The tuning range of the Cr:forsterite oscillator was measured using an spectrometer (SOLAR LASER SYSTEM, S150-II). Tuning was achieved by rotation of the mirror 25 in the horizontal plane and the results are shown in Fig. 3. For a $40 \mathrm{~mJ}$ pump energy, tunable SF output was generated from 1225 to $1275 \mathrm{~nm}$, with a tuning range of $50 \mathrm{~nm}$, peak wavelength at $1246 \mathrm{~nm}$, and a maximum output pulse energy of $1.63 \mathrm{~mJ}$ with built up time of $72 \mathrm{~ns}$.

Given the very broad amplification curve of the Cr:forsterite and that Brewster-angle related losses on the crystal faces have a negligible change per surface over $50 \mathrm{~nm}$, the system can in principle be tuned by simply tuning the oscillator in the $1222-1275 \mathrm{~nm}$ with amplifier output energy curve similar to the oscillator one. For the particular aim of our project, the system was optimized and aligned to work in a narrow region $1262 \pm 3 \mathrm{~nm}$ so the data on the amplifier performance presented here are for 
Publishingthis narrow spectral range. We note that such a tuning range of the pulse wavelength Cr:forsterite oscillator would allow to cover a broad MIR spectral region extending from 6.43 to 8.1 micron through a DFG process pumped at $1064 \mathrm{~nm}$.

To measure the linewidth of the Cr:forsterite, a second harmonic emission spectra and a configuration based on a fuse silica Fabry-Perot (FP) etalon (designed Finesse: 155.4, FRS: $10.29 \mathrm{GHz}$ ) was used. The experimental setup used for measurement of the line-width as well as the interference fringes are shown in Fig. 4a and 4b, respectively.

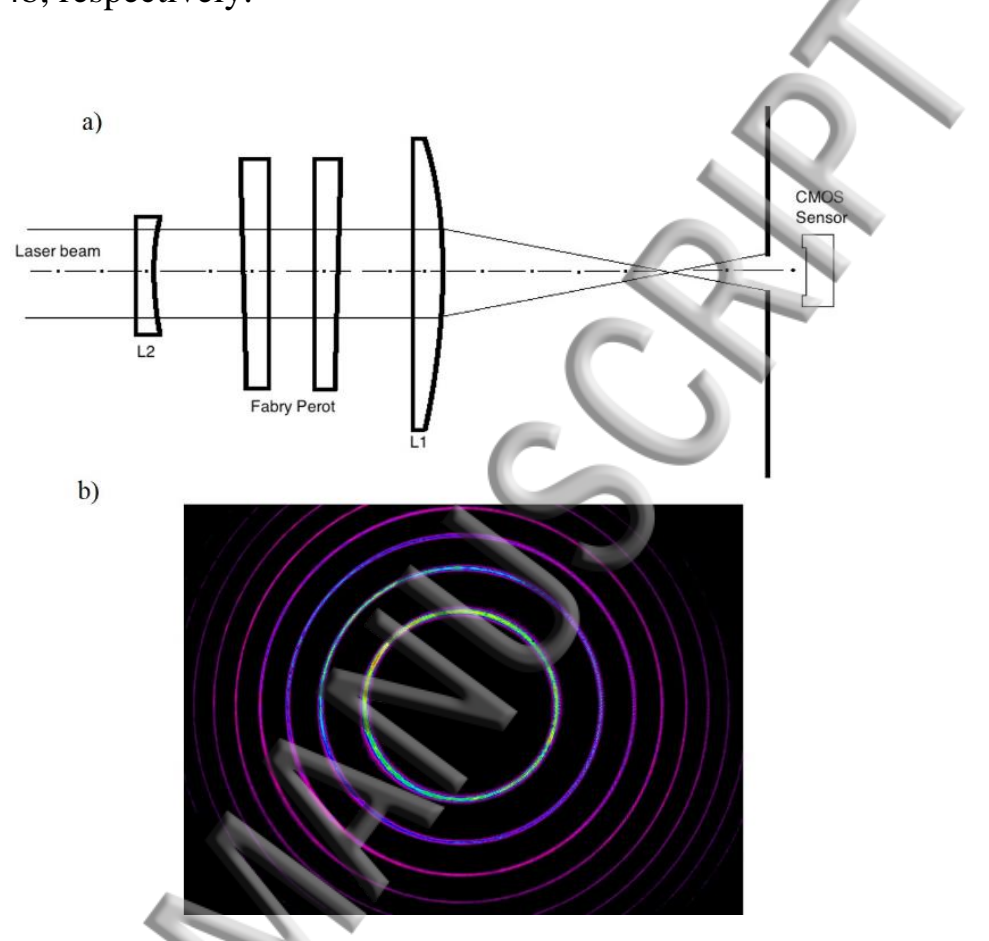

FIG. 4. Schematic of the: (a) experimental configuration and (b) the interference fringes structure used for measurement of the line-width of the oscillator.

The laser beam is expanded over the FP etalon by the divergent lens $L_{2}\left(f_{2}=-10 \mathrm{~cm}\right)$ and a second lens $\mathrm{L}_{1}\left(\mathrm{f}_{1}=25 \mathrm{~cm}\right)$ is used for imaging the fringes onto a CMOS camera (Gentec Beamage-4M). The data processing and calculation of the line-width were done using the following equation ${ }^{12}$.

$$
\Delta \lambda=\lambda \frac{r \Delta r}{f_{1}^{2}}
$$

where $\lambda=1262 \mathrm{~nm}$ is the emission wavelength, $\mathrm{r}$ is the radius of the first ring and $\Delta \mathrm{r}$ its width.

Stable SF operation of the MOPA system, with the energy stability of $2 \%$ RMS, was obtained across all the amplification stages. The linewidths were measured using an FP etalon, the calculated linewidth was $2 \mathrm{pm}$ for the oscillator and the amplifier. No increase in signal bandwidth was observed after the amplifier stages, indicating that no spectral broadening was observed, or at least that spectral broadening effects were significantly smaller than the instrument resolution. 


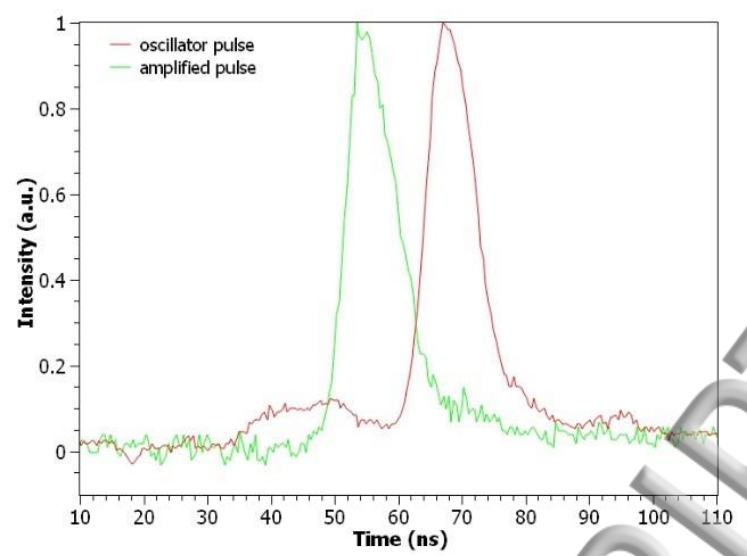

FIG. 5. Pulse shape for oscillator and after the amplifier stages.

When the initial beam is amplified in the four-stage amplifier, pulse shape distortions may arise due to the intensity dependence of the gain and because the number of passes required to reach the peak fluence also depends on the losses. To clear this point, the pulse duration and jitter were measured for both oscillator and amplifier beams, respectively. The oscillator pulse shape and the pulse after 8 passes in the amplifier are shown in Fig. 5. The pulse width was 9 ns (at FWHM) for the oscillator and the amplifier, as well as the time jitter was $\sim 2$ ns. Additionally, no differences were observed in the pulse duration and build up time as a function of the selected wavelength.

\section{CONCLUSION}

We have reported a tunable Cr:forsterite MOPA laser system based on four-stages in a two-pass configuration each. The energy has been increased 16 times with the number of stages and passes, delivering up to $24 \mathrm{~mJ}$, starting from a relatively low energy of the oscillator. The linewidth, the temporal pulse shape, as well as, the time jitter didn't change after multi-pass amplification. This system is well suited to applications, such as frequency down conversion and high-resolution muonic hydrogen spectroscopy, which requires high energy and stable SF laser operation at a particular frequency around the 6.8 micron region. It is important to note, that while the results presented here concentrate on an amplifier system operating in the relatively narrow spectral region around $1262 \mathrm{~nm}$ of interest for our application, a similar performance can be obtained in a straightforward way through the broad spectral region corresponding to the Cr:forsterite gain curve ${ }^{4}$, giving access to a very broad MIR region - 5.02 to 14.23 micron through a DFG process pumped at $1064 \mathrm{~nm}$.

\section{ACKNOWLEDGMENTS}

The authors express gratitude to INFN and Elettra for support of this work, and in particular, the SPIE-ICTP Anchor Research Program funded generously by the International Society for Optics and Photonics (SPIE). J. J. Suárez-Vargas acknowledges the funding received from the ICTP TRIL program.

${ }^{1}$ L. I. Stoychev, M. B. Danailov, A. A. Demidovich, I. P. Nikolov, P. Cinquegrana, P. Sigalotti, D. Bakalov and A. Vacchi, Proc SPIE 9135 , 91350J (2014).

${ }^{2}$ D. Bakalov, A. Adamczak, A. M. Stoilov, and A. Vacchi, Hyperfine Interact. 233, 97 (2015).

${ }^{3}$ E. Mocchiutti et al., arXiv:1905.02049.

${ }^{4}$ A. Sennaroglu, Prog. Quant. Electron. 26, 287 (2002).

${ }^{5}$ J. C. Diettrich, D. M. Warrington, and I. T. MacKinnie, Appl. Opt. 42, 2206 (2003). 
Niemela, M. Baruzzo, N. Vasiliev, Y. Zaporozhchenko, M. B. Danailov, and A. Vacchi, Laser Phys. 29, 065801 (2019).

${ }^{8}$ Q. Liu, H. Hong, X. Fu, Z. Sui, L. Huang, and M. Gong, Appl. Opt. 52, 33 (2013).

${ }^{9}$ M.G. Livshits, Y.I, Mishkel, and A. A.Tarasov, Sov. J. Quant. Elect. 19, 496 (1992).

${ }^{10}$ M. Littman and H. Metcalf, Appl. Opt. 17, 2224 (1978).

${ }^{11}$ D. J. Binks, D. K. Ko, L. A. W. Gloster, and T. A. King, J. Opt. Soc. Am. B 15, 2395 (1998).

${ }^{10}$ A. J. Tiffany, I. T. McKinnie, and D. M. Warrington, Appl. Opt. 37, 4907 (1998).

${ }^{12}$ H. G. Heard Handbook (New York: Wiley) 386 (1968). 


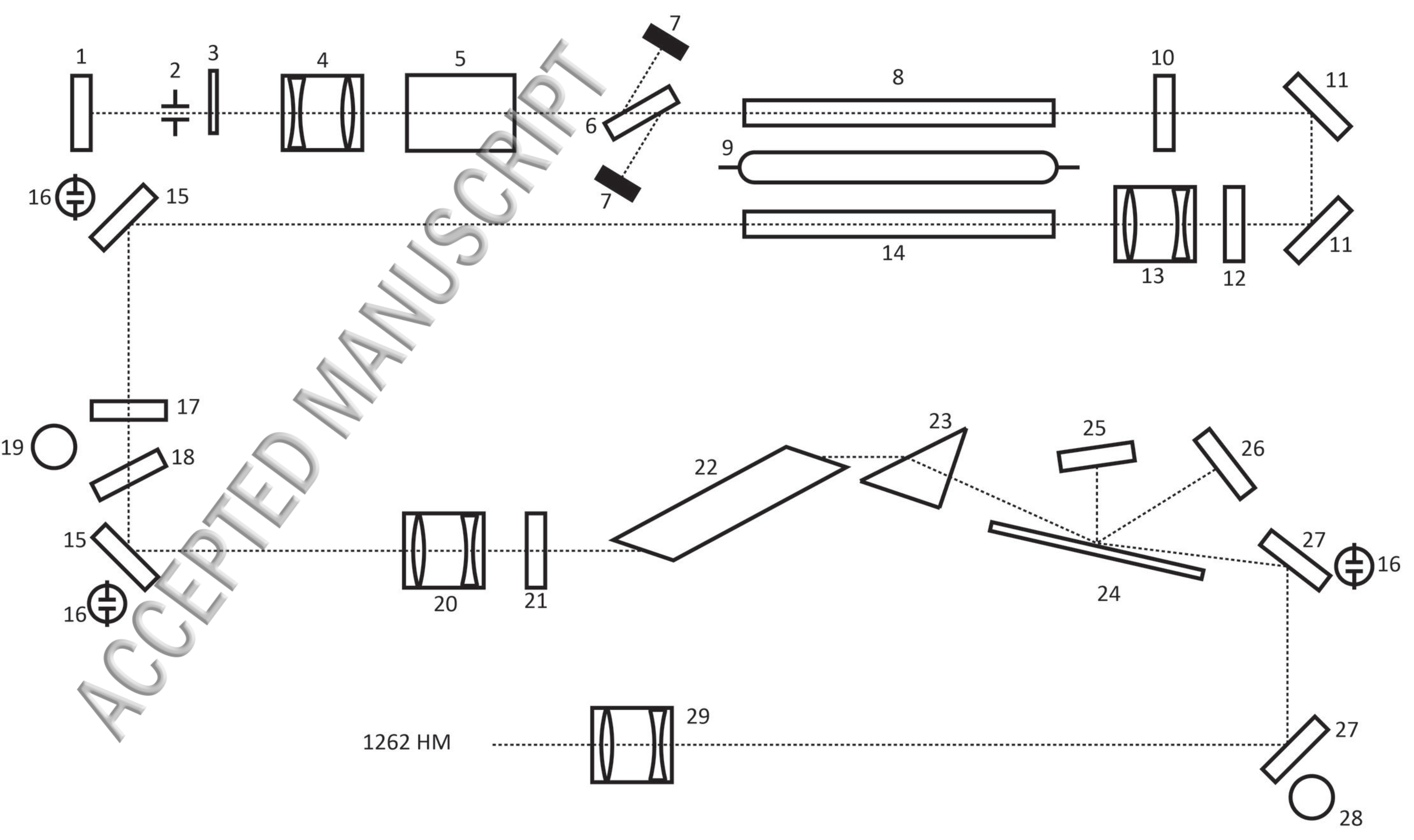




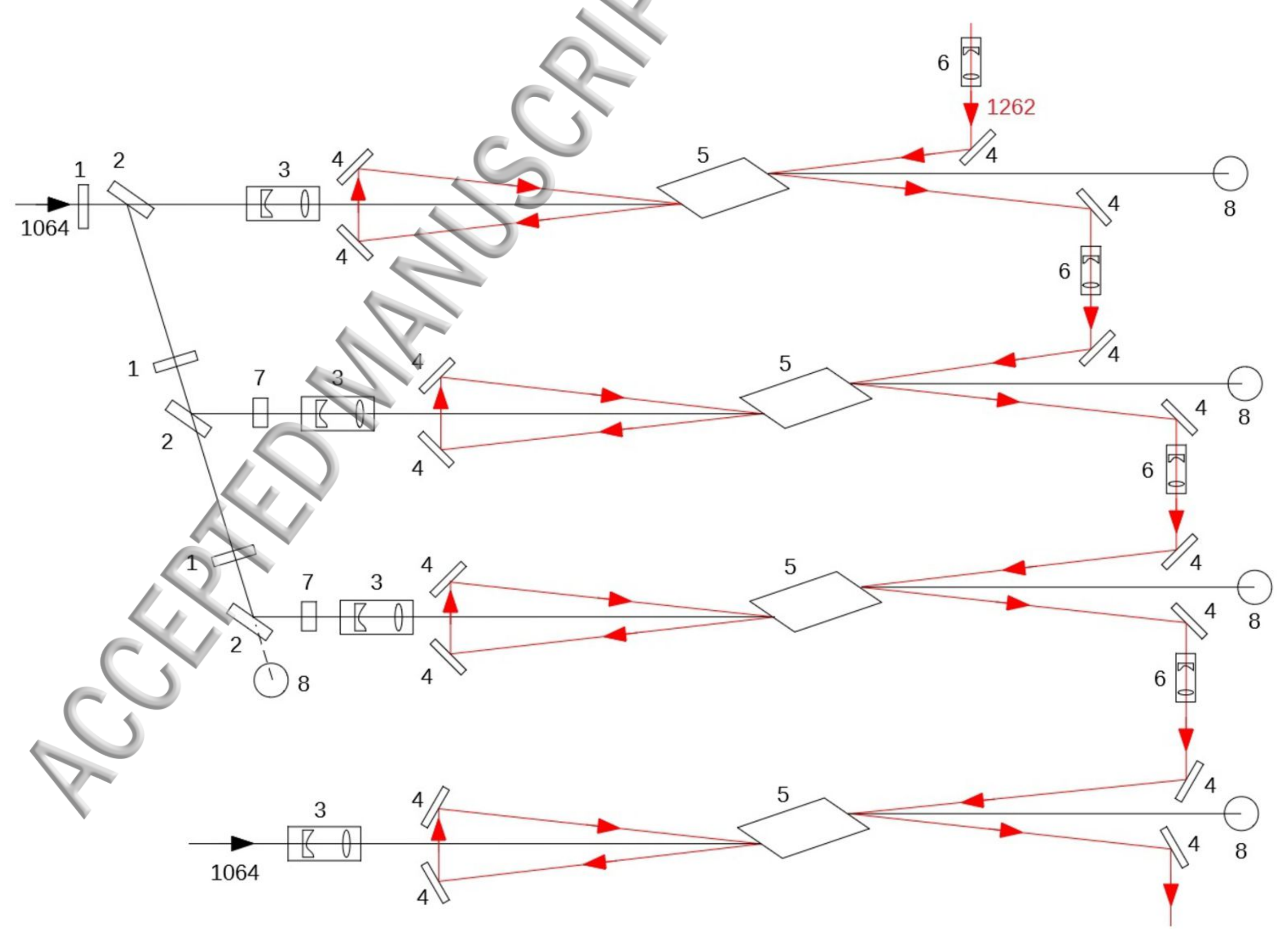




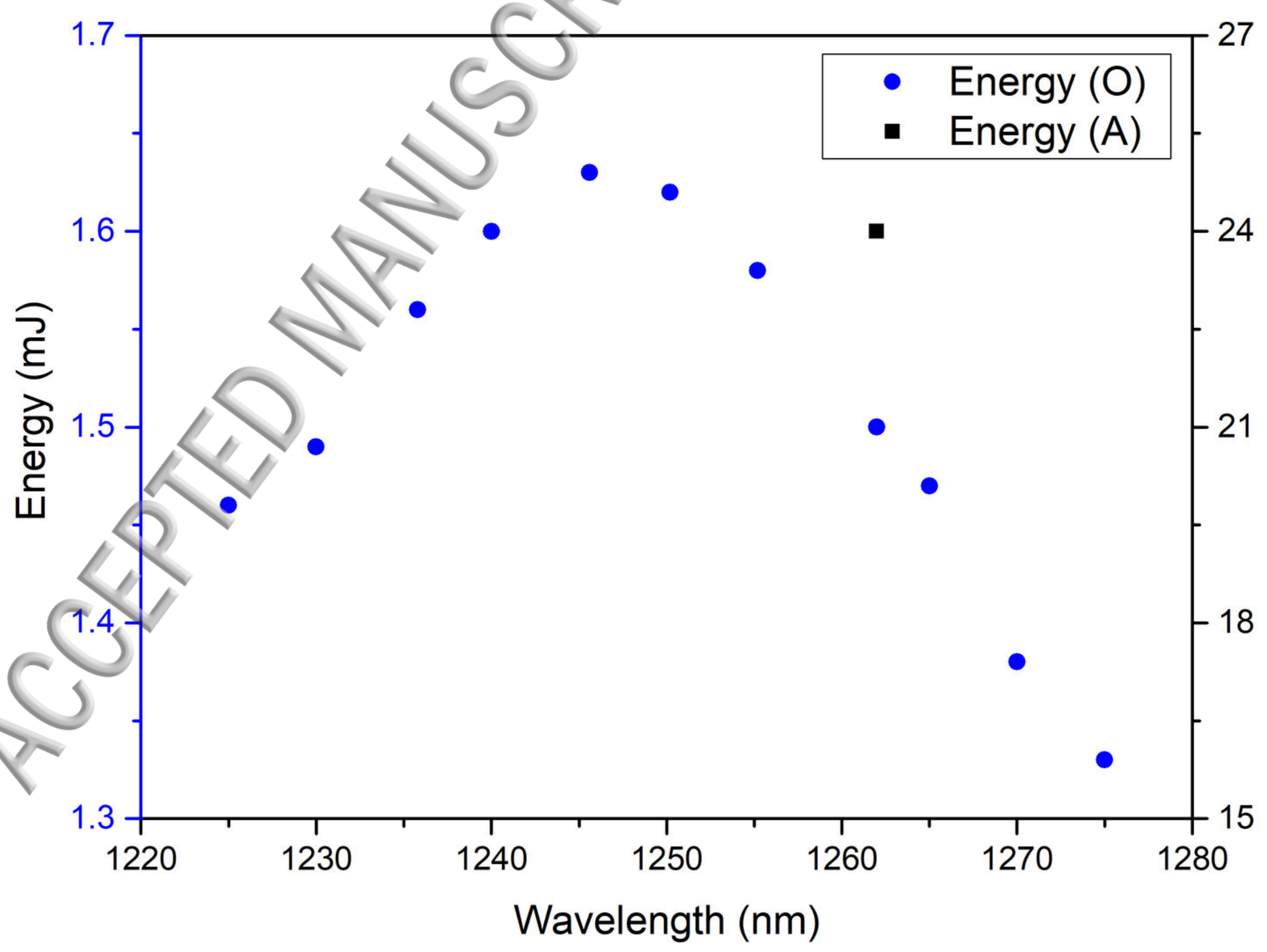




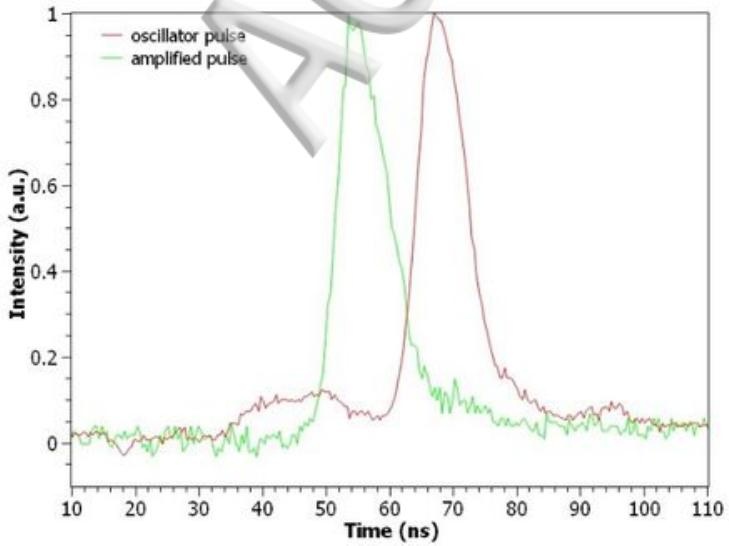

